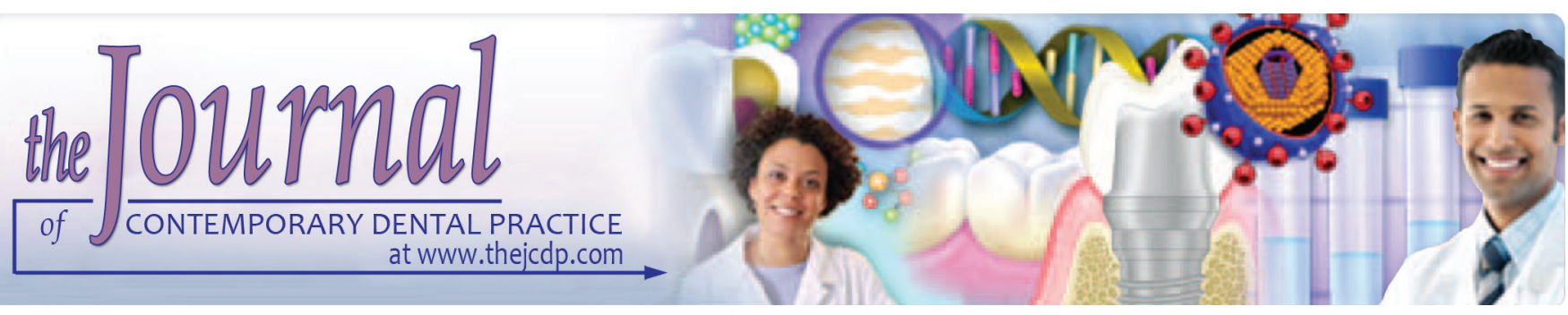

\title{
Stability of Bonded Resin Composite Restorations to Enamel after Bleaching with 20\% Carbamide Peroxide
}

Aminah M. El Mourad

\begin{abstract}
Aim: This study aimed to examine the effect of postoperative home bleaching using $20 \%$ carbamide peroxide on the shear bond strengths of different adhesives to enamel and to verify the failure mode for each test specimen.
\end{abstract}

Materials and methods: One hundred sound human molars were used in this study. Bonding procedures were performed on the flattened buccal enamel surfaces according to the manufacturer's instructions. OptiBond Solo Plus adhesive (Kerr), Single Bond Universal adhesive (3M ESPE) applied with both the total-etch and self-etch techniques, and Tetric $\mathrm{N}$-Bond Universal adhesive (Ivoclar Vivadent) applied with both the total-etch and self-etch techniques were used, followed by resin composite post fabrication (Filtek Z350 XT; 3M ESPE). All specimens were thermo-cycled for 5000 cycles and then divided into the control (non-bleach) and postoperative bleaching groups ( $20 \%$ carbamide peroxide). The shear bond strength values were measured and compared between the two groups. Data were analyzed using one-way analysis of variance with Tukey's test $(p \leq 0.05)$. The failure modes of debonded specimens were evaluated using a digital microscope (50x). The bonding interfaces of the resin composite posts to different enamel conditions (control and postoperative bleaching) were observed by a scanning electron microscope (SEM) before the shear bond test.

Results: The shear bond strength values of all tested adhesives were lower in the postoperative bleaching group than they were in the control (non-bleach) group, particularly the bond strength values of self-etch adhesives.

Conclusion: The stability of bonded resin composite restorations to enamel, using different dental adhesives, could be compromised after the bleaching procedure at varying sensitivity levels.

Clinical significance: Replacement of bonded resin composite restorations to enamel might be considered after bleaching

Department of Restorative Dental Sciences, King Saud University, Riyadh, Kingdom of Saudi Arabia

Corresponding Author: Aminah M. El Mourad, Department of Restorative Dental Sciences, King Saud University, Riyadh, Saudi Arabia, Mobile: +966 11 8055832; e-mail: amourad@ ksu.edu.sa procedures, especially if these restorations were previously bonded with self-etch adhesives.

Keywords: Bleaching, Carbamide peroxide, Resin composite, Shear bond strength.

How to cite this article: AM El Mourad. Stability of Bonded Resin Composite Restorations to Enamel after Bleaching with $20 \%$ Carbamide Peroxide. J Contemp Dent Pract 2019;20(2):247-257.

Source of support: Nil

Conflict of interest: None

\section{INTRODUCTION}

In recent years, the demand for tooth bleaching has increased because people are more concerned about improving the aesthetic appearance of their smile, and clinicians are considering more conservative, noninvasive dental treatments. Many studies in the literature have described various tooth bleaching approaches using different bleaching materials, concentrations, and lightactivation techniques. ${ }^{1}$ Dental bleaching can be divided into vital and non-vital tooth bleaching. The three main vital tooth-bleaching protocols include home bleaching, in-office bleaching, and over-the-counter bleaching agents.

Bleaching procedures were primarily performed in dental offices until Haywood and Heymann ${ }^{2}$ introduced the first home tooth bleaching agent in 1989, a $10 \%$ carbamide peroxide gel. Home tooth bleaching is advantageous because it is cost-effective, can be selfapplied by the patient, is safe, and has fewer undesirable side effects, all of which means the patient can spend less time at dental clinics. ${ }^{3}$ Tooth bleaching approaches that use oxidizing agents, such as hydrogen peroxide and carbamide peroxide, involve their circulation from the enamel to the dentin. ${ }^{4}$ Following this, the oxidizing agent disintegrates, yielding unstable free radicals. The oxidation of organic pigmented molecules is enhanced by these free radicals, leading to the formation of less heavily pigmented constituents; thus, bleaching of the tooth tissues occurs. ${ }^{5-8}$ 
Stained teeth are usually treated conservatively using bleaching procedures. However, since the bleaching material is held in intimate contact with the teeth and any associated restorations, this may cause undesirable effects on the natural tooth structure, ${ }^{9,10}$ bonding interface, and restorative materials, such as softening and degradation of the teeth and restorative materials. ${ }^{11-13}$ Thus, researching how bleaching agents react with preexisting or subsequent dental restorations is essential. Many studies have examined the reduced bond strengths of bonded restorations to enamel and dentin following tooth bleaching procedure. ${ }^{14-16}$ Other studies evaluated the effects of bleaching on pre-existing bonded restorations using a variety of methods, including bond strength measurements, ${ }^{17-20}$ fracture resistance measurements, ${ }^{21-23}$ and leakage analyses. ${ }^{24-26}$ Nevertheless, only a few studies have investigated the effect of bleaching materials on the bond strength and stability of pre-existing resin composite restorations. The stability of bonded resin composite restorations to tooth structure plays an important role in determining the durability of these restorations. The objectives of the current research were to evaluate the effect of postoperative home bleaching procedures using 20\% carbamide peroxide on the shear bond strengths of different adhesive systems to enamel and to verify the failure mode for each test specimen.

\section{Research Hypothesis}

The tested null hypothesis was that bleaching with $20 \%$ carbamide peroxide would not reduce the shear bond strength values of different adhesive systems to enamel surface and would not affect the stability of resin composite-enamel bonds.

\section{MATERIALS AND METHODS}

A total of 100 sound human molars were cleaned and kept in distilled water with a $0.05 \%$ thymol solution. The roots were cut from the teeth $2 \mathrm{~mm}$ below the cementoenamel junction with a slow-speed diamond saw (Isomet 2000; Buehler, Lake Bluff, IL, USA). The teeth were embedded in a self-cure acrylic resin that was filled in polyvinyl chloride cylindrical molds in a manner that permitted the buccal surfaces to be exposed. Flattening and polishing of the buccal surface of each tooth was performed using a series of wet 240-, followed by 400-, and then 600-grit silicon carbide paper disks (Buehler) mounted in an Automata Machine (Jeanwirtz, GMBH, West Germany) to yield a flat enamel surface. Table 1 lists all of the materials that were used in this study. The samples were randomly divided into two main groups $(n=50$ teeth per group), namely the control (non-bleach) group and the postoperative bleaching group. Each group was then subdivided into five subgroups depending on the type of adhesive utilized ( $n=10$ teeth per subgroup). The five adhesives were as follows:
- OptiBond (OB) Solo Plusadhesiveapplied (Kerr, Orange, CA, USA) using the total-etch (also known as etch-and-rinse) technique;

- Single Bond Universal adhesive (3M ESPE, Seefeld, Germany) applied using the total-etch (SB-TE) or etch-and-rinse technique; 3. Single Bond Universal (3M ESPE) adhesive applied using the self-etch (SB-SE) technique;

- Tetric N-Bond Universal adhesive (Ivoclar Vivadent, Schaan, Liechtenstein) applied using the total-etch (TNB-TE) or etch-and-rinse technique; and

- Tetric N-Bond Universal adhesive (Ivoclar Vivadent) applied using the self-etch (TNB-SE) technique.

After dental adhesive application, resin composite posts (Filtek Z350 XT; 3M ESPE Dental Products, St. Paul, MN, USA) were fabricated in each specimen, using custom-made silicone molds ( $3 \mathrm{~mm}$ in diameter, $2 \mathrm{~mm}$ high) (Fig. 1A). To ensure proper condensation of the composite, the material was placed in the tube in the silicone mold, overfilled, and then pressed with a glass slab before being light-cured for 20 seconds. Following this, the silicone mold was gently removed, and the excess composite was trimmed with a plastic instrument. Manufacturer's instructions were followed in all bonding procedures (Table 1). An Elipar S10 LED curing light (3M ESPE Dental Products) was used to perform the light-curing procedures, with a light power density of $1000 \mathrm{~mW} / \mathrm{cm}^{2}$, which was examined regularly using a Bluephase Meter II radiometer (Ivoclar Vivadent, NY, USA). Afterward, all specimens were kept in distilled water at $37^{\circ} \mathrm{C}$ for 24 hours and thermo-cycled for 5000 cycles in a thermocycling apparatus (Thermocycler 1100/1200; SD Mechatronik, Germany). ${ }^{27}$ Once the thermocycling procedures were complete, storage of specimens was done in artificial saliva at $37^{\circ} \mathrm{C}$ for 1 week. Subsequently, the specimens in the control (nonbleach) group underwent shear bond testing. For the postoperative bleaching group, a carbamide peroxide bleaching material (Opalescence PF 20\%; Ultradent ${ }^{\circledR}$ Products, Inc., South Jordan, UT, USA) was applied on each specimen's bond interface daily for 14 consecutive days to simulate the home bleaching technique (Fig. 1B). The bleaching gel was placed on the specimen each day for 4 hours; the specimens were subsequently washed under running water to remove the bleaching gel completely, followed by storage in artificial saliva at $37^{\circ}$ C. After the 14-day bleaching treatment, the specimens were subjected to shear bond testing.

The shear bond testing procedures were accomplished using a universal testing machine (Instron 5965; Instron, Norwood, MA, USA) and a load cell of $10 \mathrm{kN}$ at a crosshead speed of $0.5 \mathrm{~mm} / \mathrm{min}$.

The failure modes of the debonded specimens were analyzed using a digital microscope (KH-7700; Hirox, Hackensack, NJ, USA) at 50× magnification. The 

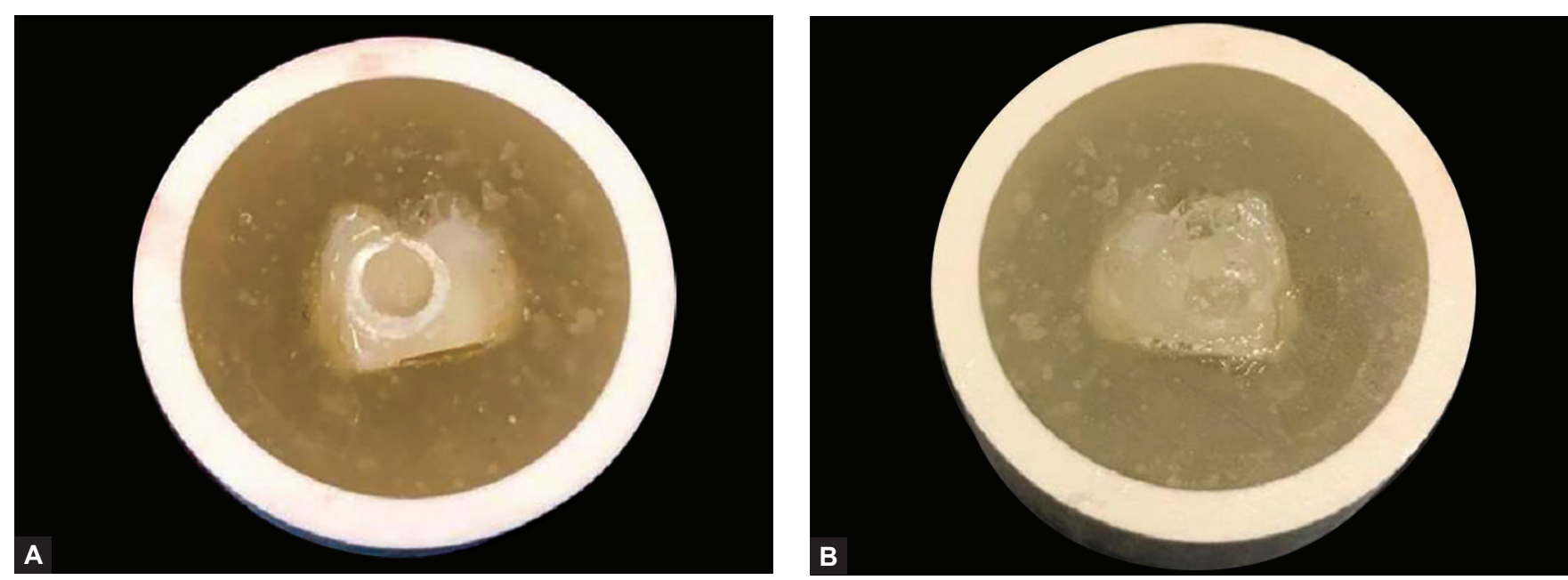

Figs 1A and B: (A) The specimen after resin composite post fabrication; (B) The specimen after bleaching gel application

Table 1: Materials used in the study

\begin{tabular}{llll}
\hline Material & Manufacturer & Chemical composition & Application technique \\
\hline Filtek & 3M ESPE Dental & Bis-GMA, UDMA, TEGDMA, & Application of the resin composite and light curing \\
Z350 XT & products, St. & Bis-EMA,20 nm nanosilica, & of each applied increment for20 s. \\
Universal & Paul, MN, USA & zirconia/silica particles &
\end{tabular}

nanocomposite resin

Opalescence PF $20 \%$

voclarVivadent
Ultra-etch acid etchant

OptiBond Solo Plus Used as

Total-etch (etch-andrinse technique)

\section{Single Bond Universal}

Used as

Total-etch (etch-andrinse) and self-etch techniques

Tetric N-Bond Universa Used as Schaan, Liechtenstein

Ultradent

Products Inc.,

South Jordan UT, USA

Kerr, Orange, CA, USA

3M ESPE, Seefeld, Germany

Total-etch (etch-andrinse) and self-etch techniques
$20 \%$ carbamide peroxide, sodium fluoride, potassium nitrate, glycerin, water (aqua), silica, sorbitol, xylitol, flavor (aroma), poloxamer, sodium lauryl sulfate, carbomer, sodium benzoate, sodium hydroxide, sparkle, sucralose, xanthan gum

$$
35 \% \text { Phosphoric acid }
$$

Bis-GMA, HEMA, GPDM, ethanol, barium, aluminium borosilicate glass, fumed silica, sodium hexafluorosilicate, photo initiator

MDP phosphate monomer, dimethacrylate resins, HEMA, Vitrebond copolymer, filler, ethanol, water, initiators, silane

HEMA,D3MA,bis-GMA, MDP, MCAP, ethanol, water, highly dispersed silicon dioxide, initiators and stabilisers
Application of the bleaching material $4 \mathrm{~h} / \mathrm{d}$ for 14 consecutive days to simulate home bleaching procedure.
Enamel is etched with the acid etchant for appearance of enamel.

Enamel is etched with the acid etchant for $15 \mathrm{~s}$; rinsed and air dried to produce a frosty appearance of enamel, then, the adhesive is applied, airthinned for $3 \mathrm{~s}$, and light cured for $20 \mathrm{~s}$.

If it is used as etch-and-rinse technique, enamel is etched with the acid etchant for $15 \mathrm{~s}$; rinsed and air dried to produce a frosty appearance of enamel, then, the adhesive is applied, air-thinned for $5 \mathrm{~s}$, and light cured for $10 \mathrm{~s}$.

If it is used as self-etch technique, the adhesive is applied directly without enamel etching technique.

If it is used as etch-and-rinse technique, enamel is etched with the acid etchant for $15 \mathrm{~s}$; rinsed and air dried to produce a frosty appearance of enamel, then, the adhesive is applied, air-thinned for $5 \mathrm{~s}$, and light cured for $10 \mathrm{~s}$.

If it is used as self-etch technique, the adhesive is applied directly without enamel etching technique.

*Statistically significant difference

fractures were assigned to one of the following categories based on the position of the fracture line: (a) Adhesive failures:fractures positioned between the adhesive material and enamel or between the adhesive material and resin composite; (b) Cohesive failures:fractures propagated through the substrates, i.e. enamel tooth tissue or resin composite; or (c) Mixed failures: mixed modes of fracture, including adhesive and cohesive fractures. 
Scanning electron microscope (SEM) (JSM-6360LV; JEOL, Tokyo, Japan) was used to observe the bonding interfaces of the resin composite posts, which were bonded using the five dental adhesives, under different enamel conditions (non-bleach and postoperative bleaching) before shear bond strength testing at $100 \times$ magnification. Before performing SEM, the specimens were dried and sputter-coated with gold (Fine Coater JFC-1200; JEOL).

The shear bond strength data were analyzed using SPSS version 21.0(IBM Corporation, Armonk, NY). The shear bond strength values are reported using descriptive statistics [mean, standard deviation (SD)]. The mean shear bond strength values of the five adhesive material subgroups within each of the two main groups (non-bleach, postoperative bleaching) were compared using one-way analysis of variance followed by Tukey's multiple comparison tests. Comparisons of the mean shear bond strength values between the non-bleach and postoperative bleaching groups for each adhesive material were conducted using Student's $t$-test. Statistical significance was set at $p \leq 0.05$.

\section{RESULTS}

The shear bond strength at the maximum load was identified for the five different adhesive materials (OB, SB-TE, SB-SE, TNB-TE, and TNB-SE) under each of the two enamel conditions (control non-bleach and postoperative bleaching groups). The descriptive statistics (mean and SD) of the shear bond strength values are presented in Table 2.

\section{Within-group Comparisons}

\section{Control Group (non-bleach)}

The mean shear bond strength values among the tested adhesive materials (OB, SB-TE, SB-SE, TNB-TE, and TNB-SE) in the control group (non-bleach) were significantly different $(\mathrm{F}=108.45, p<0.0001)$ (Table 3 and Graph 1). Tukey's multiple comparison tests between different pairs of adhesive materials demonstrated that the mean shear bond strength values of the TNB-TE and SB-TE adhesives were significantly higher than were those of the other three adhesives, whereas the mean shear bond strength value of the $\mathrm{OB}$ adhesive was significantly lower than were those of the other four adhesives (Table 4). The mean shear bond strength values of all adhesive material pairs were significantly different, except for OB vs. SB-SE and SB-SE vs.TNB-SE, which were not significantly different in terms of the mean shear bond strength value (Table 4).

\section{Postoperative Bleaching Group}

The mean shear bond strength values among the tested adhesive materials (OB, SB-TE, SB-SE, TNB-TE, and
Table 2: Shear bond strength means and SDs among tested adhesive materials in the two enamel conditions (control non-bleach and postoperative bleaching groups) (MPa)

\begin{tabular}{lllll}
\hline Group & Adhesive material & $N$ & Mean & SD \\
\hline Control group & OB & 10 & 18.8939 & 1.13092 \\
(Non-bleach) & SB-TE & 10 & 27.4972 & 1.3534 \\
& SB-SE & 10 & 19.5030 & 1.4084 \\
& TNB-TE & 10 & 28.7076 & 1.4222 \\
& TNB-SE & 10 & 21.7400 & 1.5693 \\
Post-bleach & Total & 50 & 23.2683 & 4.3269 \\
group & OB & 10 & 16.8282 & 1.9757 \\
& SB-TE & 10 & 24.4362 & 1.3874 \\
& SB-SE & 10 & 17.1534 & 1.8998 \\
& TNB-TE & 10 & 26.7203 & 2.0998 \\
& TNB-SE & 10 & 17.1640 & 1.4290 \\
& Total & 50 & 20.4604 & 4.6134 \\
\hline
\end{tabular}

Table 3: Comparison of mean shear bond strength values among the tested adhesive materials in control group (MPa)

\begin{tabular}{lllll}
\hline $\begin{array}{l}\text { Type of } \\
\text { material }\end{array}$ & Mean & SD & F-value & $p$-value \\
\hline OB & 18.8939 & 1.1309 & 108.45 & $<0.0001^{*}$ \\
SB-TE & 27.4972 & 1.3534 & & \\
SB-SE & 19.5030 & 1.4084 & & \\
TNB-TE & 28.7076 & 1.4222 & & \\
TNB-SE & 21.7400 & 1.5693 & & \\
\hline
\end{tabular}

${ }^{*}$ Statistically significant difference

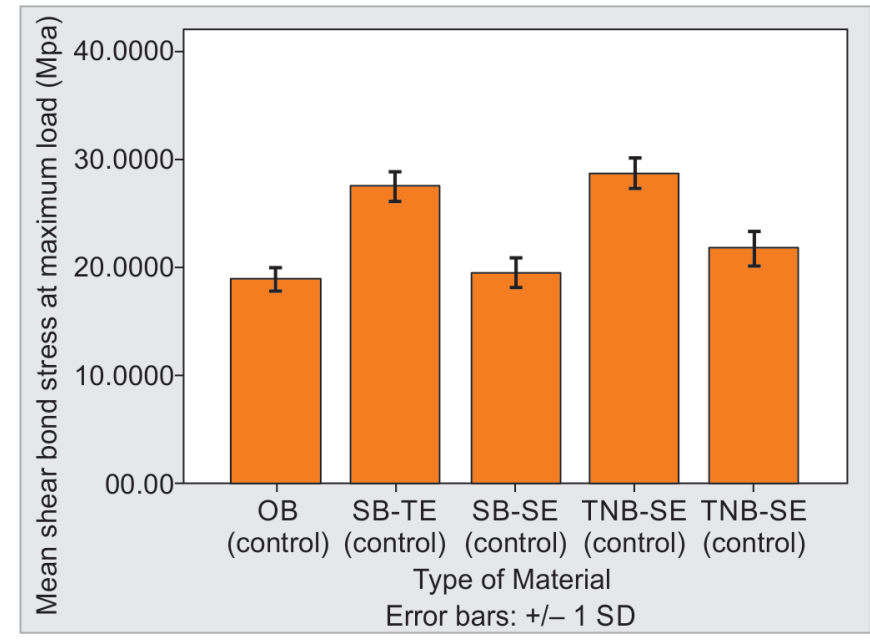

Graph 1: Comparison of mean shear bond strength values among the tested adhesive materials in control group (MPa)

TNB-SE) in the postoperative bleaching group were significantly different $(\mathrm{F}=70.791, p<0.0001)$ (Table 5 and Graph 2). Tukey's multiple comparison test between different pairs of adhesive materials demonstrated that the mean shear bond strength values of the TNB-TE and SB-TE adhesives were significantly higher than were those of the other three adhesives (OB, SB-SE, and TNB$\mathrm{SE})$, whereas the mean shear bond strength values of the OB, SB-SE, and TNB-SE adhesives were significantly lower than were those of the TNB-TE and SB-TE adhesives (Table 6). The mean shear bond strength values of all 
Stability of Bonded Composite Restorations to Enamel after Bleaching

\begin{tabular}{|c|c|c|c|c|c|}
\hline \multirow[b]{2}{*}{ (I) Material } & \multirow[b]{2}{*}{ (J) Material } & \multirow[b]{2}{*}{ Mean difference (I-J) } & \multirow[b]{2}{*}{$p$ value } & \multicolumn{2}{|c|}{$95 \%$ Confidence interval } \\
\hline & & & & Lower bound & Upper bound \\
\hline \multirow[t]{4}{*}{ OB } & SB-TE & $-8.6032^{*}$ & $<0.0001$ & -10.3621 & -6.8442 \\
\hline & SB-SE & -.6090 & .861 & -2.3679 & 1.1499 \\
\hline & TNB-TE & $-9.8136^{*}$ & $<0.0001$ & -11.5726 & -8.0547 \\
\hline & TNB-SE & $-2.8460^{*}$ & $<0.0001$ & -4.6049 & -1.0870 \\
\hline \multirow[t]{4}{*}{ SB-TE } & $\mathrm{OB}$ & $8.6032^{*}$ & $<0.0001$ & 6.8442 & 10.3621 \\
\hline & SB-SE & $7.9942^{*}$ & $<0.0001$ & 6.2352 & 9.7531 \\
\hline & TNB-TE & -1.2104 & .304 & -2.9693 & .5484 \\
\hline & TNB-SE & $5.7572^{*}$ & $<0.0001$ & 3.9982 & 7.5161 \\
\hline \multirow[t]{4}{*}{ SB-SE } & $\mathrm{OB}$ & .6090 & .861 & -1.1499 & 2.3679 \\
\hline & SB-TE & $-7.9942^{*}$ & $<0.0001$ & -9.7531 & -6.2352 \\
\hline & TNB-TE & $-9.2046^{*}$ & $<0.0001$ & -10.9635 & -7.4457 \\
\hline & TNB-SE & $-2.2370^{*}$ & .006 & -3.9959 & -.4780 \\
\hline \multirow[t]{4}{*}{ TNB-TE } & $\mathrm{OB}$ & $9.8136^{\star}$ & $<0.0001$ & 8.0547 & 11.5726 \\
\hline & SB-TE & 1.2104 & .304 & -.5484 & 2.9693 \\
\hline & SB-SE & $9.2046^{*}$ & $<0.0001$ & 7.4457 & 10.9635 \\
\hline & TNB-SE & $6.9676^{*}$ & $<0.0001$ & 5.2087 & 8.7265 \\
\hline \multirow[t]{4}{*}{ TNB-SE } & $\mathrm{OB}$ & $2.8460^{*}$ & $<0.0001$ & 1.0870 & 4.6049 \\
\hline & SB-TE & $-5.7572^{*}$ & $<0.0001$ & -7.5161 & -3.9982 \\
\hline & SB-SE & $2.2370^{\star}$ & .006 & .4780 & 3.9959 \\
\hline & TNB-TE & $-6.9676^{*}$ & $<0.0001$ & -8.7265 & -5.2087 \\
\hline
\end{tabular}

*Statistically significant difference

Table 5: Comparison of mean shear bond strength values among the tested adhesive materials in postoperative bleaching group (MPa)

\begin{tabular}{lllll}
\hline Type of material & Mean & $S D$ & F value & $p$ value \\
\hline OB & 16.8282 & 1.9757 & 70.791 & $<0.0001^{*}$ \\
SB-TE & 24.4362 & 1.3874 & & \\
SB-SE & 17.1534 & 1.8998 & & \\
TNB-TE & 26.7203 & 2.0998 & & \\
TNB-SE & 17.1640 & 1.4290 & & \\
\hline
\end{tabular}

*Statistically significant difference

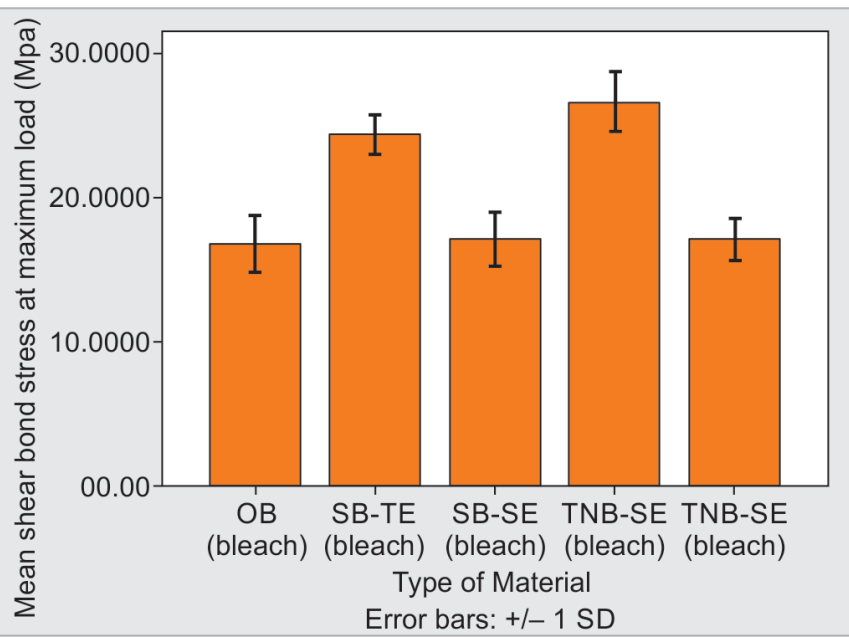

Graph 2: Comparison of mean shear bond strength values among the tested adhesive materials in postoperative bleaching group (MPa)

adhesive material pairs were significantly different except for OB vs. SB-SE, OB vs. TNB-SE, and SB-SE vs. TNB-SE, which were not significantly different in terms of the mean shear bond strength value (Table 6).
Table 6: Multiple comparison of mean shear bond strength values among the tested adhesive materials in the postoperative bleaching group (MPa)

\begin{tabular}{|c|c|c|c|c|c|}
\hline \multirow{3}{*}{ (I) } & \multirow{3}{*}{$\begin{array}{l}(J) \\
\text { Material }\end{array}$} & \multirow{3}{*}{$\begin{array}{l}\text { Mean } \\
\text { Difference } \\
(I-J)\end{array}$} & \multirow[b]{3}{*}{$p$ value } & \multicolumn{2}{|c|}{$\begin{array}{l}95 \% \text { Confidence } \\
\text { interval }\end{array}$} \\
\hline & & & & Lower & Upper \\
\hline & & & & bound & bound \\
\hline \multirow[t]{4}{*}{$\mathrm{OB}$} & SB-TE & $-7.6080^{*}$ & $<0.0001$ & -9.8733 & -5.3426 \\
\hline & SB-SE & -.3251 & .994 & -2.5904 & 1.9401 \\
\hline & TNB-TE & $-9.8920^{*}$ & $<0.0001$ & -12.1574 & -7.6267 \\
\hline & TNB-SE & -.33576 & .993 & -2.6010 & 1.9295 \\
\hline \multirow[t]{4}{*}{ SB-TE } & OB & $7.6080^{*}$ & $<0.0001$ & 5.3426 & 9.8733 \\
\hline & SB-SE & $7.2828^{*}$ & $<0.0001$ & 5.0175 & 9.5481 \\
\hline & TNB-TE & $-2.2840^{*}$ & .047 & -4.5494 & -.0187 \\
\hline & TNB-SE & $7.2722^{*}$ & $<0.0001$ & 5.0069 & 9.5375 \\
\hline \multirow[t]{4}{*}{ SB-SE } & $\mathrm{OB}$ & .3251 & .994 & -1.9401 & 2.5904 \\
\hline & SB-TE & $-7.2828^{*}$ & $<0.0001$ & -9.5481 & -5.0175 \\
\hline & TNB-TE & $-9.5669^{*}$ & $<0.0001$ & -11.8322 & -7.3015 \\
\hline & TNB-SE & -.01059 & 1.000 & -2.2759 & 2.2547 \\
\hline \multirow[t]{4}{*}{ TNB-TE } & $\mathrm{OB}$ & $9.8920^{*}$ & $<0.0001$ & 7.6267 & 12.1574 \\
\hline & SB-TE & $2.2840^{\star}$ & .047 & .01875 & 4.5494 \\
\hline & SB-SE & $9.5669^{*}$ & $<0.0001$ & 7.3015 & 11.8322 \\
\hline & TNB-SE & $9.5563^{*}$ & $<0.0001$ & 7.2909 & 11.8216 \\
\hline TNB- & $\mathrm{OB}$ & .3357 & .993 & -1.9295 & 2.6010 \\
\hline \multirow[t]{3}{*}{ SE } & SB-TE & $-7.2722^{*}$ & $<0.0001$ & -9.5375 & -5.0069 \\
\hline & SB-SE & .01059 & 1.000 & -2.2547 & 2.2759 \\
\hline & TNB-TE & $-9.5563^{*}$ & $<0.0001$ & -11.8216 & -7.2909 \\
\hline
\end{tabular}

*Statistically significant difference

\section{Between-group Comparisons}

Comparisons of the mean shear bond strength values between the control and postoperative bleaching groups for each tested adhesive material demonstrated statistically significant differences; specifically, the mean bond strength values of all five adhesives (OB, SB-TE, 
Table 7: Comparison of mean shear bond strength values between control non-bleach and post-bleach groups in each of the tested adhesive materials [Mpa]

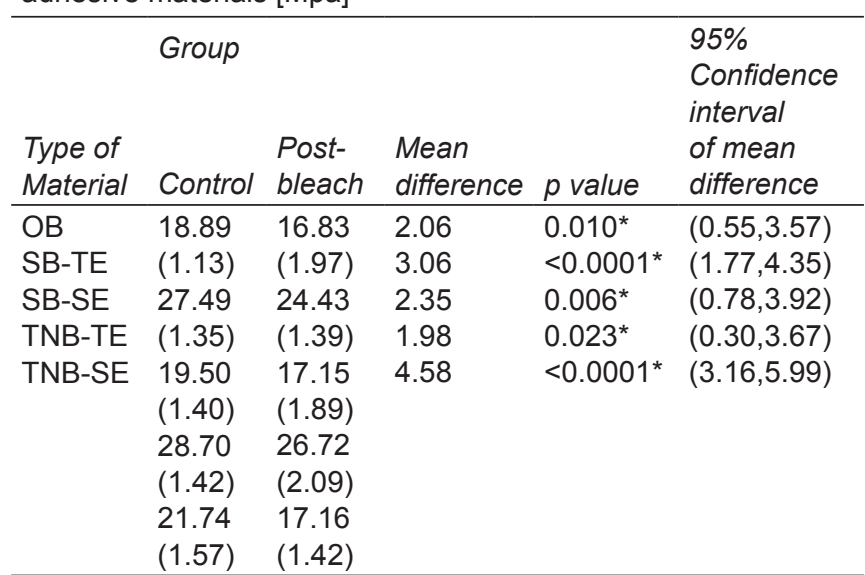

*Statistically significant difference

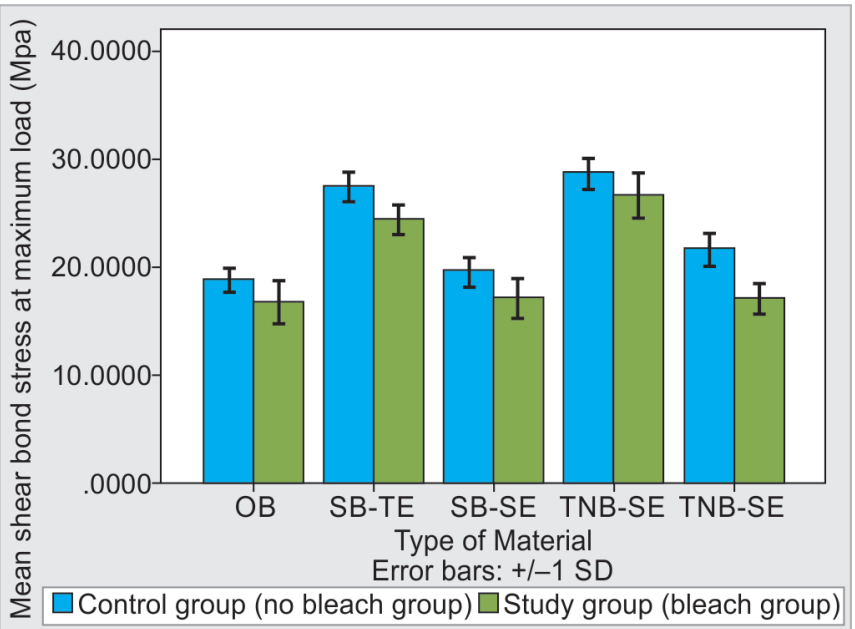

Graph 3: Comparison of mean shear bond strength values between control non-bleach and post-bleach groups in each of the tested adhesive materials (Mpa)

SB-SE, TNB-TE, and TNB-SE) were significantly lower in the postoperative bleaching group than they were in the control group (Table 7 and Graph 3). The mean difference in the shear bond strength was higher when using the TNB-SE adhesive, whereas the mean difference was lower when using the TNB-TE adhesive (Table 7 and Graph 3).

\section{Failure Analysis}

Table 8 presents the failure mode of each tested adhesive. The bleached specimens of all five tested adhesives exhibited higher adhesive failure percentages than did the corresponding unbleached specimens.

\section{Scanning Electron Microscope Testing}

The SEM analysis proved that the bond strengths of the five dental adhesives were significantly affected by the condition of the enamel. The SEM images demonstrated good contact between the resin composite posts and the
Table 8: Specimen numbers and percentages of each failure mode

\begin{tabular}{|c|c|c|c|c|c|c|}
\hline \multirow[b]{3}{*}{ Group } & \multirow{3}{*}{$\begin{array}{l}\text { Adhesive } \\
\text { material }\end{array}$} & \multicolumn{4}{|c|}{ Failure Modes } & \multirow[b]{3}{*}{ Mixed } \\
\hline & & \multicolumn{2}{|c|}{ Adhesive } & \multicolumn{2}{|c|}{ Cohesive } & \\
\hline & & $A E$ & $A R$ & $C E$ & $C R$ & \\
\hline \multirow{5}{*}{$\begin{array}{l}\text { Control } \\
\text { non- } \\
\text { bleach } \\
\text { group }\end{array}$} & $\mathrm{OB}$ & 0 & $\begin{array}{l}4 \\
(40 \%)\end{array}$ & 0 & 0 & $\begin{array}{l}6 \\
(60 \%)\end{array}$ \\
\hline & SB-TE & 0 & 0 & 0 & 0 & $\begin{array}{l}10 \\
(100 \%)\end{array}$ \\
\hline & SB-SE & 0 & $\begin{array}{l}3 \\
(30 \%)\end{array}$ & 0 & 0 & $\begin{array}{l}7 \\
(70 \%)\end{array}$ \\
\hline & TNB-TE & 0 & 0 & 0 & $\begin{array}{l}1 \\
(10 \%)\end{array}$ & $\begin{array}{l}9 \\
(90 \%)\end{array}$ \\
\hline & TNB-SE & 0 & $\begin{array}{l}1 \\
(10 \%)\end{array}$ & 0 & 0 & $\begin{array}{l}9 \\
(90 \%)\end{array}$ \\
\hline \multirow{5}{*}{$\begin{array}{l}\text { Post- } \\
\text { bleach } \\
\text { group }\end{array}$} & OB & 0 & $\begin{array}{l}8 \\
(80 \%)\end{array}$ & 0 & 0 & $\begin{array}{l}2 \\
(20 \%)\end{array}$ \\
\hline & SB-TE & $\begin{array}{l}1 \\
(10 \%)\end{array}$ & $\begin{array}{l}6 \\
(60 \%)\end{array}$ & 0 & $\begin{array}{l}1 \\
(10 \%)\end{array}$ & $\begin{array}{l}2 \\
(20 \%)\end{array}$ \\
\hline & SB-SE & $\begin{array}{l}6 \\
(60 \%)\end{array}$ & $\begin{array}{l}2 \\
(20 \%)\end{array}$ & 0 & 0 & $\begin{array}{l}2 \\
(20 \%)\end{array}$ \\
\hline & TNB-TE & $\begin{array}{l}1 \\
(10 \%)\end{array}$ & $\begin{array}{l}6 \\
(60 \%)\end{array}$ & 0 & 0 & $\begin{array}{l}3 \\
(30 \%)\end{array}$ \\
\hline & TNB-SE & $\begin{array}{l}3 \\
(30 \%)\end{array}$ & $\begin{array}{l}7 \\
(70 \%)\end{array}$ & 0 & 0 & 0 \\
\hline
\end{tabular}

(AE) Adhesive failure between the adhesive and enamel

(AR) Adhesive failure between the adhesive and resin composite

(CE) Cohesive failure in ename

(CR) Cohesive failure in resin composite

unbleached specimens, along with well-defined and distinct interfaces(Figs 2A, 3A, 4A, 5A, and 6A). The bonded interface of the resin composite posts with the same tested adhesives exhibited poor adaptation in the bleached specimens, and gaps and irregular interface margins were observed (Figs 2B, 3B, 4B, 5B, and 6B).

\section{DISCUSSION}

The current research tested the effect of postoperative home bleaching using 20\% carbamide peroxide on the shear bond strengths of different adhesives to enamel. The results showed that the bleaching procedure reduced the mean bond strength values of all five tested dental adhesives (OB, SB-TE, SB-SE, TNB-TE, and TNB-SE) and reduced the stability of resin composite-enamel bonds. Therefore, the main hypothesis of this study, stating that bleaching with $20 \%$ carbamide peroxide would not reduce the shear bond strength values of different adhesive systems to enamel surface and would not affect the stability of resin composite-enamel bonds, was rejected.

Since the introduction of dental bleaching, the use of bleaching agents for whitening discolored teeth has become more popular. In this study, the home bleaching protocol was performed using $20 \%$ carbamide peroxide. ${ }^{3}$ This concentration was used because it is considered a safe and effective home bleaching treatment. ${ }^{5}$ However, dental bleaching agents must come in close contact with 

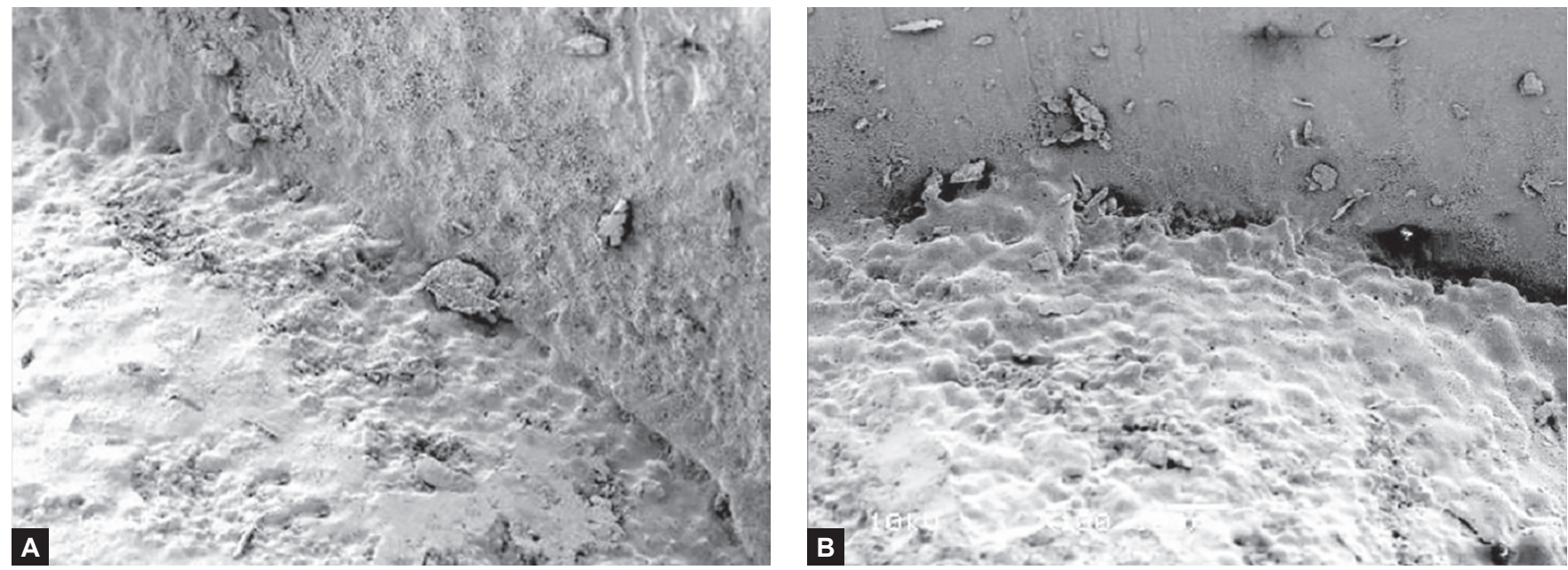

Figs $2 A$ and $B$ : Scanning electron microscope images $(\times 100)$ of the bonding interface of resin composite post with OptiBond Solo Plus under different enamel conditions: (A) Control non-bleach group; (B) Postoperative bleaching group
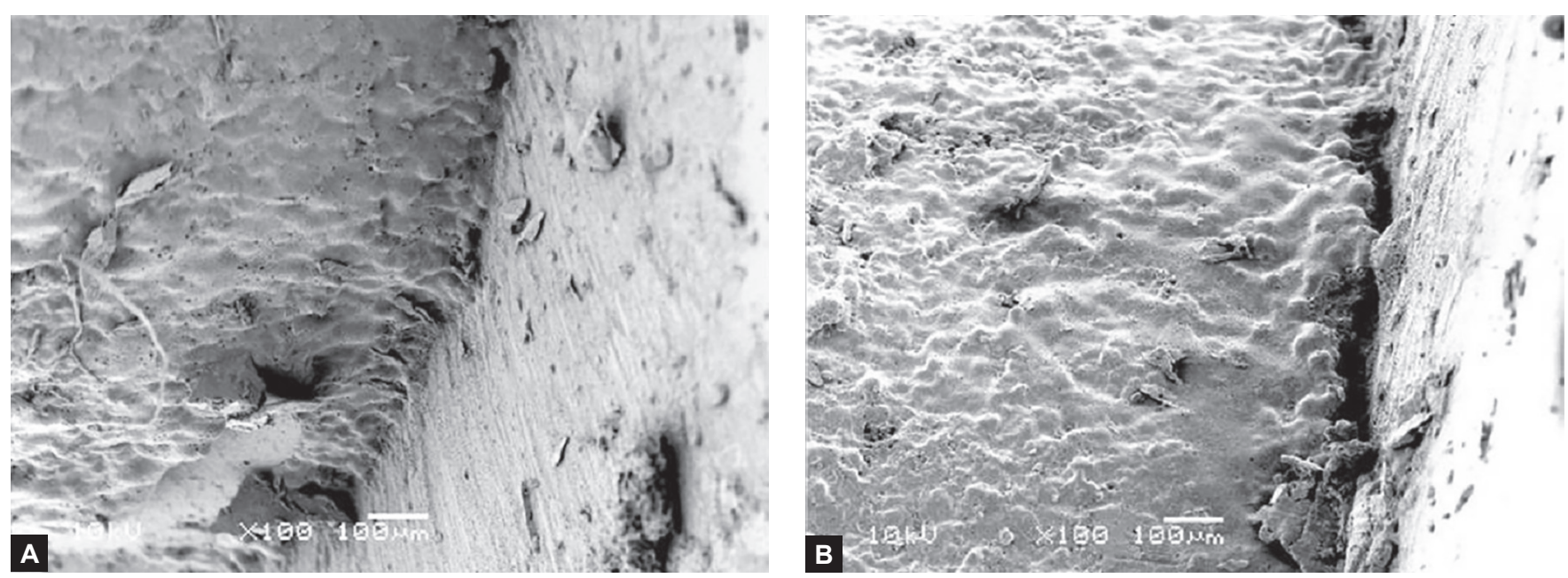

Figs 3 A and B: Scanning electron microscope images $(\times 100)$ of the bonding interface of resin composite post with Single Bond Universal adhesive applied using total-etch technique under different enamel conditions: (A) Control non-bleach group; (B) Postoperative bleaching group
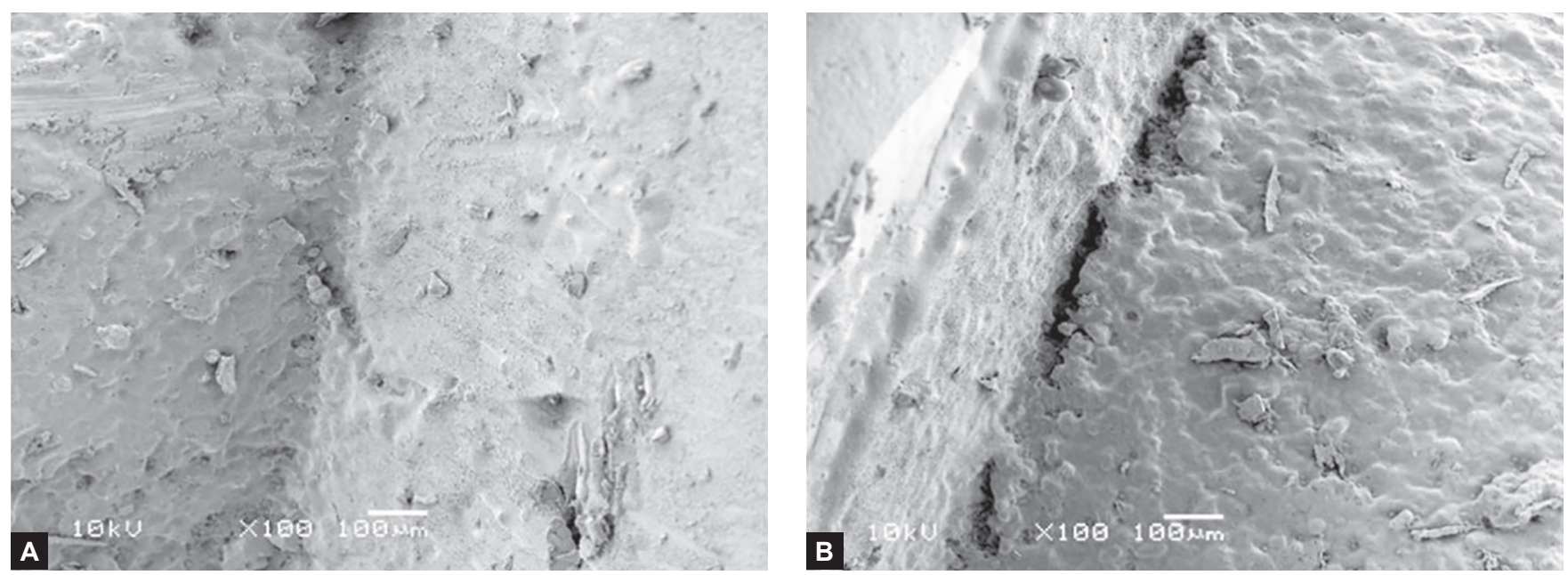

Figs 4A and B: Scanning electron microscope images $(\times 100)$ of the bonding interface of resin composite post with Single Bond Universal adhesive applied using self-etch technique under different enamel conditions: (A) Control non-bleach group; (B) Postoperative bleaching group 


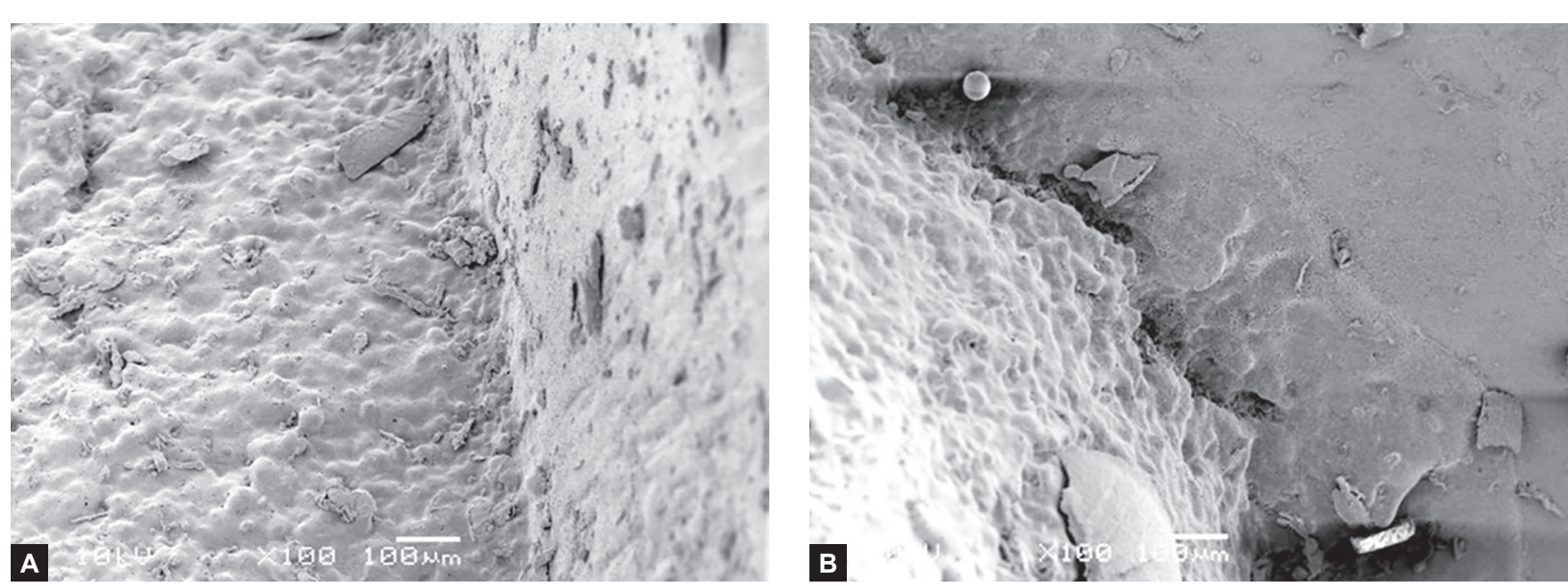

Figs 5A and B: Scanning electron microscope images $(\times 100)$ of the bonding interface of resin composite post with Tetric N-Bond Universal adhesive applied using total-etch technique under different enamel conditions: (A) Control non-bleach group, (B) Postoperative bleaching group
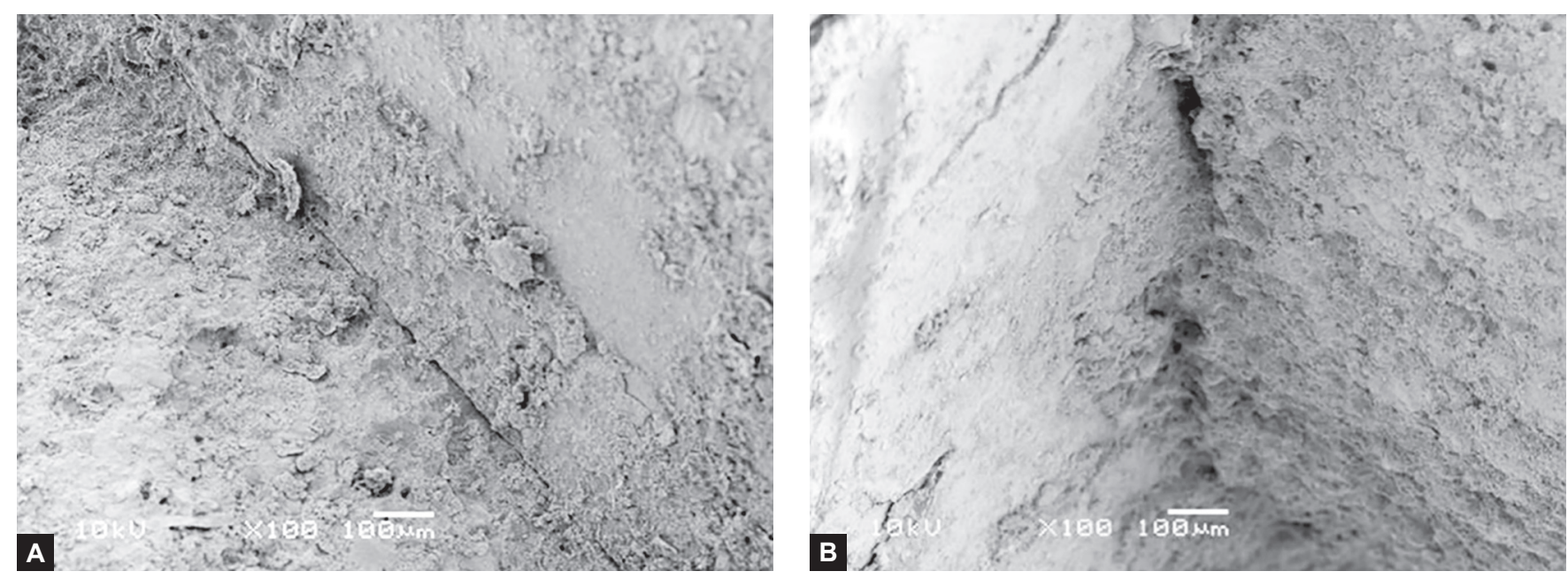

Figs 6 A and B: Scanning electron microscope images $(\times 100)$ of the bonding interface of resin composite post with Tetric N-Bond Universal adhesive applied using self-etch technique under different enamel conditions: (A) Control non-bleach group; (B) Postoperative bleaching group

the tooth surface and must penetrate and react with the dental substrate. This reaction is not specific, and, because bleaching products contact the teeth and dental restorations for extended periods of time, especially during home bleaching treatments, the bleaching agents may cause undesirable effects on both the tooth structure and restorative materials. ${ }^{4}$ The current study demonstrated that bleaching significantly reduced the shear bond strength values of the five tested adhesive materials when compared with the values of the unbleached specimens.

Universal adhesives are one of the most recent innovations in adhesive dentistry. These products are also called multi-mode or multi-purpose adhesives because they can be applied using the TE or SE approach. ${ }^{28,29}$ A variety of studies have tested the bonding efficiency of these materials on dental substrates, the results of which suggest that the performance of these universal adhesives is materialdependent. ${ }^{30-32}$ In the present study, two universal adhesives were used, namely the SB and TNB adhesives. Both adhesives were applied using the TE and SE techniques. The results of the current study revealed that the mean bond strength values of TE adhesives (TNB-TE and SB-TE) on unbleached enamel were significantly higher than were those of SE adhesives (TNB-SE and SB-SE) and the OB adhesive, with no significant differences in the bond strength values between the TNB-TE and SB-TE adhesives or between the OB and SB-SE adhesives. These results agree with those of many previous studies reporting that stable resin composite bonding to enamel was achieved using etch-and-rinse adhesives and less stable resin composite bonding to enamel attained with selfetch ones. ${ }^{19,33-36}$ However, in contrast to our study, other studies did not identify a difference in the bond strength to enamel between TE and SE adhesives. ${ }^{37-39}$ The findings 
of the current study can be attributed to the unique chemical composition of universal adhesives and the use of the TE technique. When using the SB adhesive, chemical bonding is achieved between the acidic methacryloyloxydecyl dihydrogen phosphate (MDP) and enamel, which permits the formation of stable and durable interfaces, good mechanical properties, and an improved rate of conversion of its hydrophobic resin. ${ }^{40,41}$ Therefore, the presence of MDP in the SBadhesive may underlie the higher associated bond strength. ${ }^{42}$ The chemical composition of TNB is based on a combination of hydrophilic hydroxyethyl methacrylate (HEMA), hydrophobic decanediol dimethacrylate (D3MA), intermediate bisphenol A glycidyl methacrylate (bisGMA)monomers, MDP, and methacrylated carboxylic acid polymer (MCAP). ${ }^{42}$ This combination of properties permits the TNB adhesive to dependably bridge the gap between the hydrophilic tooth structure and the hydrophobic restorative resin material under various surface conditions. ${ }^{42}$ Moreover, both universal adhesives showed better bond strengths when the enamel surface was pre-treated with phosphoric acid. This finding agrees with the findings of many previous studies showing that applying acid etch in conjunction with SE adhesive systems, mainly in the enamel, is important. ${ }^{30,31,43-46}$ The bond strength relies mainly on the adhesive's capability to demineralize the smear layer and the underlying tooth structure. ${ }^{47}$ Both the TNB-TE and SB-TE adhesives showed high bond strength values to unbleached and bleached enamel, likely owing to properly etched enamel surfaces and to the many micro-retentions that are achieved with phosphoric acid etching. Phosphoric acid pre-treatment increases the enamel roughness and removes the superficial layer of enamel, and as such, the enamel is more receptive to the SE systems, which are usually less capable than TE systems of dissolving the smear layer and etching the enamel surface. ${ }^{48}$ Therefore, both of the universal adhesives evaluated in this study (SB and TNB) exhibited high bond strength values to unbleached and bleached enamel when applied using TE technique.

Here, the bond strength values for the $\mathrm{OB}$ adhesive were not significantly different from those for the SB-SE adhesive in the control (non-bleach) and postoperative bleaching groups. This finding was consistent with those of another study, which reported similar bond strength values for the $\mathrm{OB}$ and SB-SE adhesives to bleached enamel. ${ }^{17}$

In general, the SEM analysis displayed good contact between the resin composite posts and the unbleached control specimens, along with well-defined and distinct interfaces, for all five tested dental adhesives. The major failure mode for all of the tested adhesives in the control group was the mixed type. Both SEM testing and failure analysis findings for the control group can be attributed to the stable and reliable bond strength of the tested adhesives, especially TNB-TE and SB-TE.

All of the tested adhesives in this study, including the TE adhesives, exhibited lower mean shear bond strength values to enamel in the bleached vs.unbleached specimens, particularly theSE adhesives. This is in accordance with the results of other studies that reported a significant decrease in bond strength values of both TE and SE adhesives following bleaching procedures. ${ }^{18,19}$ These findings can be explained by the fact that peroxide radicals in the bleaching materials usually penetrate the bonded interface margins, resulting in deteriorated adhesive joints and unpredictable bond strengths to enamel, especially with SE adhesives with no pre-existing stable adhesive bond strength. This detrimental effect of peroxide radicals on adhesive bonds is reported to increase with higher concentrations of carbamide peroxide..$^{18}$ On the other hand, the results of the current study disagree with the results of other studies demonstrating that bleaching treatment significantly decreased the bond strength values of SE adhesives but not of TE adhesives to enamel. ${ }^{17,20}$ The discrepant results might be explained by the use of higher concentration of bleaching material in the current and the other consistent studies which could have caused damaging effects on both TE and SE adhesives, particularly on the SE adhesives. ${ }^{18,19}$

The effects of bleaching were confirmed in the SEM analysis, as the bonded interface of the resin composite posts exhibited poor adaptation with all tested adhesives in the bleached vs. unbleached specimens, and gaps and irregular interface margins were observed in the bleached specimens. The major failure mode in the postoperative bleaching group for all tested adhesives was an adhesive failure. Both SEM testing and failure analysis findings for the postoperative group indicated that the bonded interface was the weakened zone of the adhesive bond and was the most vulnerable to deterioration by the bleaching process.

\section{CONCLUSION}

Higher concentration of bleaching agents may significantly deteriorate the bond strength of resin composite restorations; therefore, using a lower concentration of these agents might guarantee less detrimental effects on the adhesive joints.

The stability of bonded resin composite restorations to enamel, using different dental adhesives, could be compromised after the bleaching procedure at varying sensitivity levels. 
It is advisable to pre-treat the enamel with phosphoric acid etching when using SE adhesives to ensure better bond strength values.

Further investigations are needed to confirm the results obtained from this research and to test the bond strength of other adhesives following dental bleaching.

\section{CLINICAL SIGNIFICANCE}

Replacement of bonded resin composite restorations to enamel might be considered after bleaching procedures, especially if these restorations were previously bonded with SE adhesives.

\section{REFERENCES}

1. Calderini A, Sciara S, Semeria C, Pantaleo G, Polizzi E. Comparative clinical and psychosocial benefits of tooth bleaching: different light activation of a $38 \%$ peroxide gel in a preliminary case-control study. Clin Case Rep. 2016;4(8):728-735.

2. Haywood VB, Heymann HO. Nightguard vital bleaching. Quintessence Int. 1989;20(3):173-176.

3. Alqahtani MQ. Tooth-bleaching procedures and their controversial effects: A literature review. Saudi Dent J. 2014;26(2): 33-46.

4. Cavalli V, Silva BG, Berger SB, Abuna G, Marson FC, Tabchoury $\mathrm{C}$, et al. Effect of adhesive restoration and bleaching technique on the concentration of hydrogen peroxide in the pulp chamber. Oper Dent. 2017;42(2):E44-E54.

5. Haywood VB. History, safety, and effectiveness of current bleaching techniques and applications of the nightguard vital bleaching technique. Quintessence Int. 1992;23(7):471-88.

6. Joiner A. The bleaching of teeth: a review of the literature. J Dent. 2006;34(7):412-419.

7. Minoux M, Serfaty R. Vital tooth bleaching: biologic adverse effects-a review. Quintessence Int. 2008;39(8):645-659.

8. Dahl JE, Pallesen U. Tooth bleaching--a critical review of the biological aspects. Crit Rev Oral Biol Med. 2003;14(4):292-304.

9. Elfallah HM, Bertassoni LE, Charadram N, Rathsam C, Swain MV. Effect of tooth bleaching agents on protein content and mechanical properties of dental enamel. Acta Biomater. 2015; 20:120-128.

10. Menezes RP, Silva PD, Leal PC, Faria ESAL. Impact of $35 \%$ hydrogen peroxide on color and translucency changes in enamel and dentin. Braz Dent J. 2018;29(1):88-92.

11. Yu H, Zhang CY, Wang YN, Cheng H. Hydrogen peroxide bleaching induces changes in the physical properties of dental restorative materials: Effects of study protocols. J EsthetRestor Dent. 2018;30(2):E52-E60.

12. Hannig C, Duong S, Becker K, Brunner E, Kahler E, Attin T. Effect of bleaching on subsurface micro-hardness of composite and a polyacid modified composite. Dent Mater. 2007;23(2):198-203.

13. Turker SB, Biskin T. Effect of three bleaching agents on the surface properties of three different esthetic restorative materials. J Prosthet Dent. 2003;89(5):466-473.

14. Ismail EH, Kilinc E, Hardigan PC, Rothrock JK, Thompson JY, Garcia-Godoy C.Effect of two-minute application of 35\% sodium ascorbate on composite bond strength following bleaching. J Contemp Dent Pract. 2017;18(10):874-880.
15. Briso AL, Rahal V, Sundfeld RH, dos Santos PH, Alexandre RS. Effect of sodium ascorbate on dentin bonding after two bleaching techniques. Oper Dent. 2014;39(2):195-203.

16. Danesh-Sani SA, Esmaili M. Effect of $10 \%$ sodium ascorbate hydrogel and delayed bonding on shear bond strength of composite resin and resin-modified glass ionomer to bleached enamel.J Conserv Dent. 2011;14(3):241-246.

17. Elmourad A, Alqahtani M. Effects of pre- and post-simulated home bleaching with $10 \%$ carbamide peroxide on the shear bond strengths of different adhesives to enamel. The Saudi Journal for Dental Research. 2014;5(2):81-92.

18. Barcellos DC, Benetti P, Fernandes VV, Jr., Valera MC. Effect of carbamide peroxide bleaching gel concentration on the bond strength of dental substrates and resin composite. Oper Dent. 2010;35(4):463-469.

19. Dudek M, Roubickova A, Comba L, Housova D, Bradna P. Effect of postoperative peroxide bleaching on the stability of composite to enamel and dentin bonds. Oper Dent. 2013;38(4):394-407.

20. Cavalli V, de Carvalho RM, Giannini M. Influence of carbamide peroxide-based bleaching agents on the bond strength of resin-enamel/dentin interfaces. Braz Oral Res. 2005;19(1):23-29.

21. Bagheri R, Fani M, Barfi Ghasrodashti A, Nouri Yadkouri N Mousavi S. Effect of a home bleaching agent on the fracture toughness of resin composites, using short rod design. J Dent (Shiraz). 2014;15(2):74-80.

22. Far C, Ruse ND. Effect of bleaching on fracture toughness of composite-dentin bonds. J Adhes Dent. 2003;5(3):175-182.

23. Woo JM, Ho S, Tam LE. The effect of bleaching time on dentin fracture toughness in vitro. J EsthetRestor Dent. 2010;22(3):179-184.

24. Piemjai M, Lenglerdphol S. Effect of strong tooth-bleaching with $38 \%$ hydrogen peroxide on marginal seal of dental restorations using self-etch and total-etch adhesives. J EsthetRestor Dent. 2018;30(2):153-159.

25. Klein CA, Jr., da Silva D, Reston EG, Borghetti DL, Zimmer R. Effect of at-home and in-office bleaching on marginal microleakage in composite resin restorations using two adhesive systems. J Contemp Dent Pract. 2018;19(3):248-252.

26. Mortazavi V, Fathi M, Soltani F. Effect of postoperative bleaching on microleakage of etch-and-rinse and self-etch adhesives. Dent Res J (Isfahan). 2011;8(1):16-21.

27. Burke FJ, Hussain A, Nolan L, Fleming GJ. Methods used in dentine bonding tests: an analysis of 102 investigations on bond strength. Eur J ProsthodontRestor Dent. 2008;16(4): 158-165.

28. Hanabusa M, Mine A, Kuboki T, Momoi Y, Van Ende A, Van Meerbeek B, et al. Bonding effectiveness of a new 'multi-mode' adhesive to enamel and dentine. J Dent. 2012;40(6):475-484.

29. Perdigao J, Sezinando A, Monteiro PC. Laboratory bonding ability of a multi-purpose dentin adhesive. Am J Dent. 2012;25(3):153-158.

30. McLean DE, Meyers EJ, Guillory VL, Vandewalle KS. Enamel bond strength of new universal adhesive bonding agents. Oper Dent. 2015;40(4):410-417.

31. Perdigao J, Loguercio AD. Universal or multi-mode adhesives: why and how? J Adhes Dent. 2014;16(2):193-194.

32. Perdigao J, Munoz MA, Sezinando A, Luque-Martinez IV, Staichak R, Reis A, et al. Immediate adhesive properties to 
dentin and enamel of a universal adhesive associated with a hydrophobic resin coat. Oper Dent. 2014;39(5):489-499.

33. Perdigao J, Gomes G, Gondo R, Fundingsland JW. In vitro bonding performance of all-in-one adhesives. Part I-microtensile bond strengths. J Adhes Dent. 2006;8(6):367-373.

34. Carvalho RM, Manso AP, Geraldeli S, Tay FR, Pashley DH. Durability of bonds and clinical success of adhesive restorations. Dent Mater. 2012;28(1):72-86.

35. Kerby RE, Knobloch LA. Microtensile bond strength of one-step and self-etching adhesive systems. Oper Dent. 2005;30(2):195-200.

36. Pashley DH, Tay FR. Aggressiveness of contemporary selfetching adhesives Part II. Effects on unground enamel. Dent Mater. 2001;17(5):430-440.

37. Tay FR, Pashley DH, King NM, Carvalho RM, Tsai J, Lai SC, Marquezini L., Jr Aggressiveness of self-etch adhesives on unground enamel. Oper Dent. 2004;29(3):309-316.

38. Perdigao J, Lopes MM, Lambrechts P, Leitao J, Van Meerbeek B, Vanharle G. Effects of a self-etching primer on enamel shear bond strengths and SEM morphology. Am J Dent. 1997;10(3):141-146.

39. Shimada Y, Senawongse P, Harnirattisai MF, Nakaoki BY, Tagami J. Bond strength of two adhesive systems to primary and permanent enamel. Oper Dent. 2002;27(4):403-409.

40. de Lima E, Santos R, Durao M, Nascimento A, Braz R. Universal cements: dual activated and chemically activated. Acta BiomaterOdontol Scand. 2016;2(1):125-129.
41. Yazdi FM, Moosavi H, Atai M, Zeynali M. Dentin bond strength and degree of conversion evaluation of experimental self-etch adhesive systems. J Clin Exp Dent. 2015;7(2):e243-249.

42. Jayasheel A, Niranjan N, Pamidi H, Suryakanth MB. Comparative evaluation of shear bond strength of universal dental adhesives -an in vitro study. J Clin Exp Dent. 2017;9(7):e892-e896.

43. Poggio C, Scribante A, Della Zoppa F, Colombo M, Beltrami $R$, Chiesa M. Shear bond strength of one-step self-etch adhesives to enamel: effect of acid pretreatment. Dent Traumatol. 2014;30(1):43-48.

44. Altunsoy M, Botsali MS, Sari T, Onat H. Effect of different surface treatments on the microtensile bond strength of two self-adhesive flowable composites. Lasers Med Sci. 2015;30(6):1667-1673.

45. Shafiei F, Saadat M. Micromorphology and bond strength evaluation of adhesive interface of a self-adhering flowable composite resin-dentin: Effect of surface treatment. Microsc Res Tech. 2016;79(5):403-407.

46. Devarasa GM, Subba Reddy VV, Chaitra NL, Swarna YM. Self-etching adhesive on intact enamel, with and without pre-etching. Microsc Res Tech. 2012;75(5):650-654.

47. Miyazaki M, Tsujimoto A, Tsubota K, Takamizawa T, Kurokawa H, Platt JA. Important compositional characteristics in the clinical use of adhesive systems. J Oral Sci. 2014;56(1):1-9.

48. Gandhi G, Kalra JPS, Goyal A, Sharma A. Microphotographic assessment of enamel surface using self-etching primer and conventional phosphoric acid: an in vitro study. Contemp Clin Dent. 2018;9(1):15-19. 